\title{
The operating performance of a biotrickling filter with Lysinibacillus fusiformis for the removal of high-loading gaseous chlorobenzene
}

\author{
Zhao-Xia Li • Bai-Ren Yang • Jian-Xiang Jin • \\ Yi-Chen Pu $\cdot$ Cheng Ding
}

Received: 20 January 2014 / Accepted: 15 May 2014/Published online: 15 June 2014

(C) The Author(s) 2014. This article is published with open access at Springerlink.com

\begin{abstract}
Removal of gaseous chlorobenzene (CB) by a biotrickling filter (BTF) filled with modified ceramics and multi-surface hollow balls during gasliquid mass transfer at the steady state was by microbial degradation rather than dissolution in the spray liquid or emission into the atmosphere. The BTF was flexible and resistant to the acid environment of the spray liquid, with the caveat that the spray liquid should be replaced once every 6-7 days. The BTF, loaded with Lysinibacillus fusiformis, performed well for purification of high-loading $\mathrm{CB}$ gas. The maximum $\mathrm{CB}$ gas inlet loading rate, $103 \mathrm{~g} \mathrm{~m}^{-3} \mathrm{~h}^{-1}$, CB elimination capacity, $97 \mathrm{~g} \mathrm{~m}^{-3} \mathrm{~h}^{-1}$, and $\mathrm{CB}$ removal efficiency, $97.7 \%$, were reached at a spray liquid flow rate of $27.6 \mathrm{ml} \mathrm{min}{ }^{-1}$, an initial CB concentration of up to $1,300 \mathrm{mg} \mathrm{m}^{-3}$, and an empty bed retention time of more than $45 \mathrm{~s}$.
\end{abstract}

Electronic supplementary material The online version of this article (doi:10.1007/s10529-014-1559-5) contains supplementary material, which is available to authorized users.

Z.-X. Li · Y.-C. Pu

School of Chemical and Biological Engineering,

Yancheng Institute of Technology, YanCheng 224051,

People's Republic of China

B.-R. Yang · J.-X. Jin · C. Ding $(\bowtie)$

School of Environmental Science and Engineering,

Yancheng Institute of Technology, Yinbin Road 9,

YanCheng 224051, People's Republic of China

e-mail: ycdingc@163.com
Keywords Biotrickling filter · Biofilms . Chlorobenzene - Gaseous chlorobenzene · Gas-liquid mass transfer $\cdot$ Lysinibacillus fusiformis
Abbreviations
BTF Biotrickling filter
CB Chlorobenzene
$C_{\text {in }} \quad$ The CB gas inlet concentration
$C_{\text {out }} \quad$ The CB gas outlet concentration
EBRT The empty bed retention time
EC The CB elimination capacity
ILR The CB gas inlet loading rate
$Q \quad$ The CB gas inlet flow rate
$R E \quad$ The CB removal efficiency
VOCs Volatile organic compounds
$v \quad$ The spray liquid flow rate

\section{Introduction}

Prolonged exposure to chlorobenzene (CB) contamination has mutagenic, teratogenic and carcinogenic effects on human health (Field and Sierra-Alvarez 2008). Therefore, the control and treatment of CB pollutants is important. The conventional treatment methods for CB pollutants include physical, chemical and biological processes. Physical and chemical processes, such as adsorption (Liu et al. 2011; Zhao et al. 2001), condensation (Huang et al. 2013), and photolytic degradation (Zhang and 
Anderson 2013), etc., often require strict operating conditions, a certain dose of other chemicals, and complex response configurations. Therefore, biological methods have become the focus of research efforts due to their low cost, simple operation, and low secondary pollution. Compared with biofilters and bioscrubbers, biotrickling filters (BTFs) offer flexible control of the spray liquid, $\mathrm{pH}$, and intermediate toxic products, and have obvious advantages in the degradation of inorganic waste gases, such as ammonia (Lopez et al. 2013; Xue et al. 2010) or a variety of volatile organic compounds (VOCs) (Nicolella et al. 2009; Lebrero et al. 2012). However, the application of a BTF to $\mathrm{CB}$ containing waste gases has been rarely reported and basic data related to this process is lacking.

Microorganisms are the key factors that determine whether BTF systems are running well (Yang et al. 2010). The common microbial agents used in the BTF include: a single dominant species, mixed species, or decomposer communities of single- and mixed-species. Under the different process conditions, each type of agent displays different degradation efficiencies. At present, the research into the predominant strains that degrade VOCs gives preference to artificially domesticated strains and mostly focuses on bacteria.

The choice of packing materials in the BTF is also crucial (Liu and Wang 2012). Packing materials with high robustness, high porosity, large specific surface area, good hydrophilicity, high surface roughness, and moderate grain size are the most suitable for microbial attachment and gasliquid mass transfer, and have advantages in resisting any drop in pressure.

In this report, one dominant high concentration CB-degrading strain, Lysinibacillus fusiformis LW13 (Li et al. 2013), was activated and cultured for amplification. It was then used to form biofilms on the packing materials in the BTF. During the stable operation of the BTF, the accumulation of intermediates and $\mathrm{pH}$ changes in the spray fluid were monitored and analyzed. The $\mathrm{CB}$ gas outlet concentration $\left(C_{\text {out }}\right)$, the $\mathrm{CB}$ gas inlet loading rate $(I L R)$, the $\mathrm{CB}$ elimination capacity $(E C)$, and the $\mathrm{CB}$ removal efficiency $(R E)$ were evaluated while varying the spray liquid flow rate $(v)$, the $\mathrm{CB}$ gas inlet concentration $\left(C_{\text {in }}\right)$, the $\mathrm{CB}$ gas inlet flow rate $(Q)$, and the empty bed retention time (EBRT).
These results will provide a starting point for future in-depth studies, and industrial applications, of CB waste gas removal by a single dominant species in the BTF.

\section{Materials and methods}

Materials

The packing material of the BTF was a mixture of modified ceramics, $1.2 \mathrm{~cm} \times 1.5 \mathrm{~cm}$, and multi-faceted hollow balls, diam. $1.5 \mathrm{~cm}$. The two packing materials were inert to chlorobenzene (CB) absorption and were randomly mixed at a ratio of modified ceramics: multi-surface hollow balls $=1-1.5: 1$. The inoculated strain, Lysinibacillus fusiformis LW13 (GenBank accession number JN166076), was prescreened with a high concentration of $\mathrm{CB}$, and its ability to use $\mathrm{CB}$ as its sole carbon source was preserved in our laboratory. The sterilized mineral medium without the carbon source (Li et al. 2013) was used as the spray liquid and prepared before use.

Schematic diagram and operation of the BTF

The BTF was made of Plexiglass, diam. 10; $120 \mathrm{~cm}$ (Fig. 1). The packing layer (total ht $80 \mathrm{~cm}$; total volume 6.281 ) was divided by five porous clapboards into four semi-continuous separate units. The diam. of each pore was $0.8 \mathrm{~cm}$ and the pores were uniformly aligned on each clapboard at intervals of $0.4 \mathrm{~cm}$. A rotation axis was inserted through the clapboards at the center and rotated at $40 \mathrm{rpm}$ to ensure good gas-liquid mass transfer. The spray liquid, sealed in a water recirculation tank, was pumped through a peristaltic pump to the top of the BTF in a countercurrent operation and was then evenly sprayed through a sprinkler on the surface of the packing materials. The liquid CB was sealed to prevent evaporative losses to the atmosphere and boiled in a water-bath along with driving of an air flow from the air compressor to form CB gas, which flowed through a rotometer and mixed with another air flow from the air compressor to obtain the simulated waste gas. The CB loading could be controlled over an appropriate range by varying the ratio of the gas flow rate of the two rotometers. The tests were carried out under atmospheric pressure at $25 \pm 2{ }^{\circ} \mathrm{C}$. $Q$ was $0.25-0.6 \mathrm{~m}^{3} \mathrm{~h}^{-1}, \quad C_{\text {in }}$ was 
Fig. 1 Schematic diagram of the biotrickling filter (BTF)

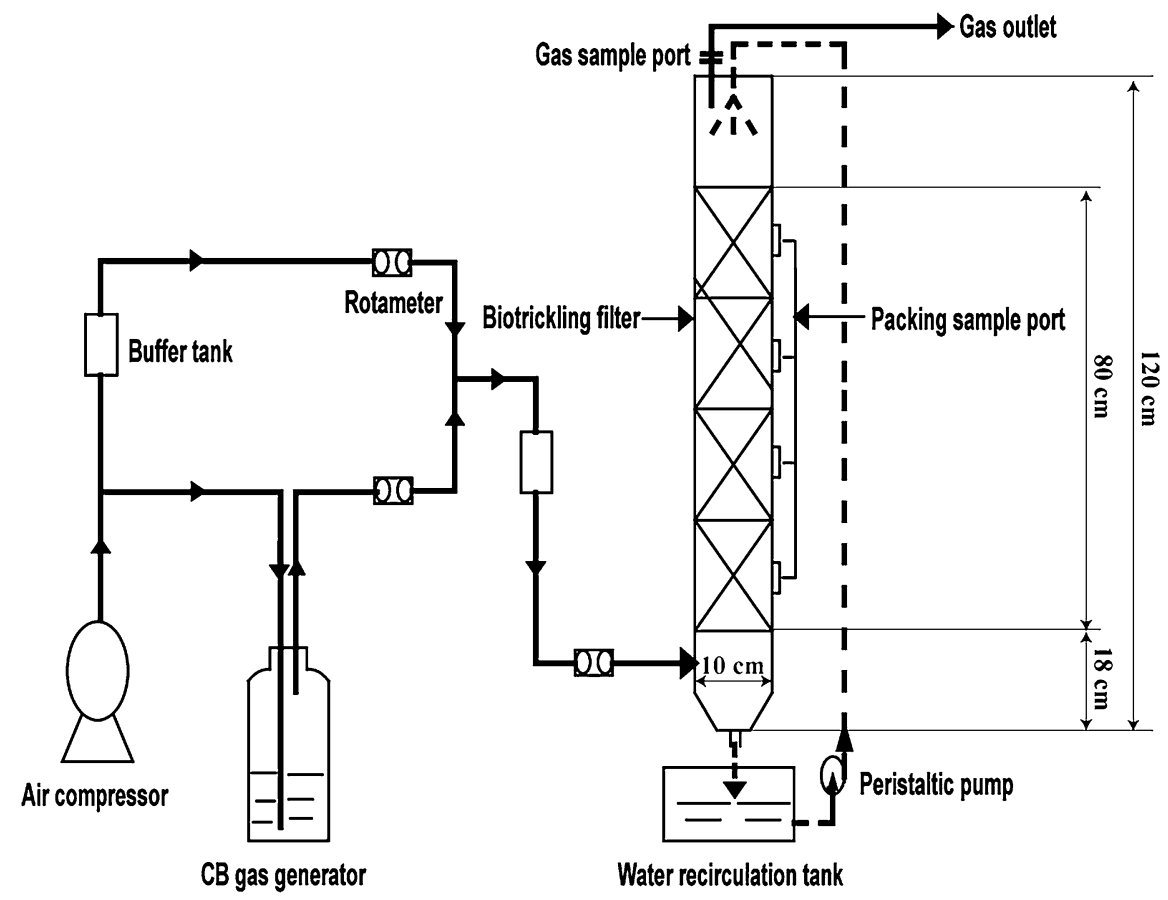

277-1,670 $\mathrm{mg} \mathrm{m}^{-3}$, and $v$ was $7.88-47.4 \mathrm{ml} \mathrm{min}^{-1}$, the corresponding EBRT was 37-90 s, and ILR was $15.7-146.18 \mathrm{~g} \mathrm{~m}^{-3} \mathrm{~h}^{-1}$. The spray liquid was refreshed once every cycle period during a $7 \mathrm{~d}$ cycle.

\section{Determination of the CB concentration}

The CB concentration was determined using headspace GC. Three parallel determinations were made for each sample and the average value was used. The peak area of the samples' standard curve was a linear function of the $\mathrm{CB}$ concentration. An Elite-5 capillary column $(30 \mathrm{~m} \times 0.32 \mathrm{~mm} \times 0.5 \mu \mathrm{m})$ was used. The volume of the splitless injection was $500 \mu \mathrm{l}$, and it was injected into the vaporization chamber at $200{ }^{\circ} \mathrm{C}$. The column flow was $1.5 \mathrm{ml} \mathrm{N}_{2} \mathrm{~min}^{-1}$ and the column temperature program was: $70^{\circ} \mathrm{C}$ for $1 \mathrm{~min}$, an increase to $110{ }^{\circ} \mathrm{C}$ at $10^{\circ} \mathrm{C} \mathrm{min}^{-1}$, and a hold at $110{ }^{\circ} \mathrm{C}$ for $1 \mathrm{~min}$. The detector (FID) was at $250{ }^{\circ} \mathrm{C}$.

Determination of $\mathrm{pH}$ value and accumulation of metabolites

The $\mathrm{pH}$ of the spray liquid was measured with a $\mathrm{pH}$ meter. The accumulation of metabolites was evaluated at $A_{255 n m}$ (Seignez et al. 2002).

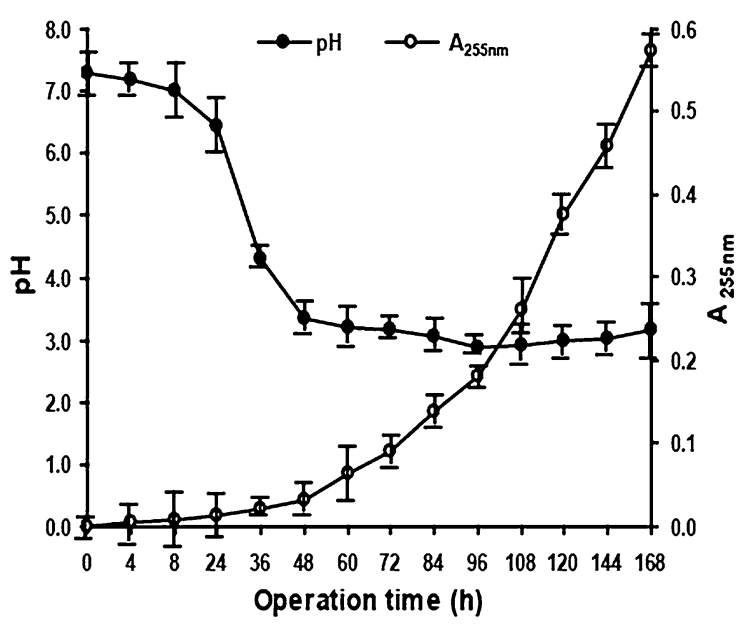

Fig. $2 \mathrm{pH}$ and $\mathrm{A}_{255 \mathrm{~nm}}$ in the BTF within a cycle period

\section{Results and discussion}

$\mathrm{pH}$ and intermediate products in the BTF within a cycle period

In the BTF, the dominant degradation strain produces $\mathrm{HCl}$ during the $\mathrm{CB}$ biodegradation process causing the system to become acidic, which can, in turn, affect the CB levels. CB biodegradation also produces a variety 
of metabolites, the accumulation of which can affect growth and even have a toxic effect on the dominant degradation strain. The BTF was run for one cycle under conditions of a $v$ of $30 \mathrm{ml} \mathrm{min}^{-1}, C_{\text {in }}$ of $1,200 \mathrm{mg} \mathrm{m}^{-3}$, and an EBRT of $75 \mathrm{~s}$ from a corresponding $Q$ of $0.3 \mathrm{~m}^{3} \mathrm{~h}^{-1}$. To examine the effect on the $\mathrm{CB}$ levels as a function of $\mathrm{pH}$ value and the accumulation of metabolites, the $\mathrm{pH}$ value of the spray liquid and its $A_{255 n m}$ (Seignez et al. 2002) were monitored over time (Fig. 2).

Within $60 \mathrm{~h}$, the $\mathrm{pH}$ of the spray liquid decreased rapidly from an initial $\mathrm{pH}$ of 7.3 to $\mathrm{pH} 3.2$. It then fluctuated around $\mathrm{pH}$ 3. This demonstrated that the spray liquid became acidic and that the microorganisms in the BTF could adapt quickly and resist the acidic environment of the spray liquid. The $\mathrm{A}_{255 \mathrm{~nm}}$ of the spray liquid could not be detected until $96 \mathrm{~h}$, and then constantly increased to 0.574 at $168 \mathrm{~h}$. This indicated that, as shown in Supplementary Fig. 1, the metabolites gradually accumulated as the BTF was in operation and were negatively related to the $\mathrm{CB}$ purifying effect. Therefore, in the $\mathrm{CB}$ purification process, the $\mathrm{pH}$ of the spray liquid should not be adjusted as an attempt to maintain neutral conditions. Rather, to maintain and maximize the utility of the nutrients in the spray liquid, it should be replaced once every 6-7 days.

Amount of CB in the spray liquid and BTF within a cycle period

It is possible that a small amount of $\mathrm{CB}$ could be removed from the BTF by the spray liquid when the liquid is replaced. To determine this, the CB concentration in the spray liquid was continuously monitored within a cycle period, at the same time points when the $\mathrm{pH}$ and intermediate products in the BTF were determined. The results showed that the amount of $\mathrm{CB}$ in the spray liquid averaged $78 \mathrm{mg}$ with only minor fluctuations after being in operation for 2 days (Fig. 3). This was well below the average cumulative reduction of $\mathrm{CB}$, which was $40.4 \mathrm{~g}$ in the BTF in a single cycle. This illustrates that there were small levels of soluble and accumulated $\mathrm{CB}$ in the spray liquid. $C_{\text {out }}$, shown in Supplementary Fig. 1a, indicated that the average CB loss from the gas outlet to the atmosphere was $3.2 \mathrm{~g}$. Thus, the removal of $\mathrm{CB}$ was mostly due to the biological function of the dominant CB-

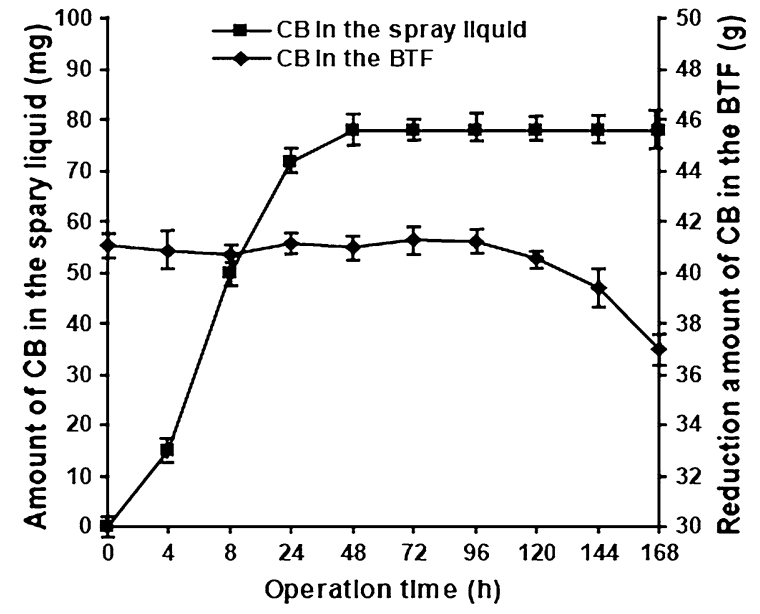

Fig. 3 Amount of chlorobenzene in the spray liquid and the BTF within a cycle period

degradation strain. Microstructures of the packing materials with biofilms formed by the dominant CB-degradation strain (Supplementary Fig. 2) and the CB levels (Supplementary Fig. 1) also demonstrated that the BTF system was at a steady operational state.

\section{CB levels as affected by $\mathrm{C}_{\mathrm{in}}$}

As CB was the sole carbon source in the BTF, $C_{\text {in }}$ would play a key role in normal microbial growth and metabolism. When $v$ was maintained at $30 \mathrm{ml} \mathrm{min}^{-1}$, the removal of CB was investigated at different $C_{\text {in }}$ of $0.25,0.4$, and $0.6 \mathrm{~m}^{3} \mathrm{~h}^{-1}$ corresponding to EBRTs of 90, 56, and 37 s, respectively (Figs. 4, 5).

At a constant $v$ and increasing $C_{\text {in }}$, at a number of different $Q$ or EBRTs, there were consistent trends in $C_{\text {out }}, I L R, E C$ and $R E$. When $C_{\text {in }}$ was increased, $C_{\text {out }}, I L R$ and $E C$ gradually increased, but $R E$ decreased significantly. This indicates that $C_{\text {in }}$ had a significant impact on the $\mathrm{CB}$ levels. Because of the reduction in the biodegradation capacity, the biodegradability of $\mathrm{CB}$ in the BTF decreased and the increase in $C_{\text {out }}$ was larger than the change in $C_{\text {in }}$. Therefore, $E C$ was negatively correlated with $R E$. Thus, the CB levels could not be evaluated by simply using just $E C$ or $R E$. Because of the assumption that $C_{\text {out }}$ had to meet the integrated emission standard of air pollutants of China (CAIES), $C_{\text {in }}$ was kept under $1,300 \mathrm{mg} \mathrm{m}^{-3}$ to improve $E C$ and $R E$ within this constraint. 
Fig. $4 C_{\text {out }}$ and $I L R$ at different $C_{\text {in }}$ and EBRTs. $C_{\text {out }}=$ chlorobenzene (CB) gas outlet concentration; $I L R$ is the $\mathrm{CB}$ gas inlet loading rate; $C_{\text {in }}$ is the $\mathrm{CB}$ gas inlet concentration; EBRT is the empty bed retention time
Fig. $5 R E$ and $E C$ at different $C_{\text {in }}$ and EBRTs.

$R E=$ chlorobenzene $(\mathrm{CB})$ removal efficiency;

$E C=\mathrm{CB}$ elimination capacity; $C_{\mathrm{in}}=\mathrm{CB}$ gas inlet concentration;

EBRT = empty bed retention time
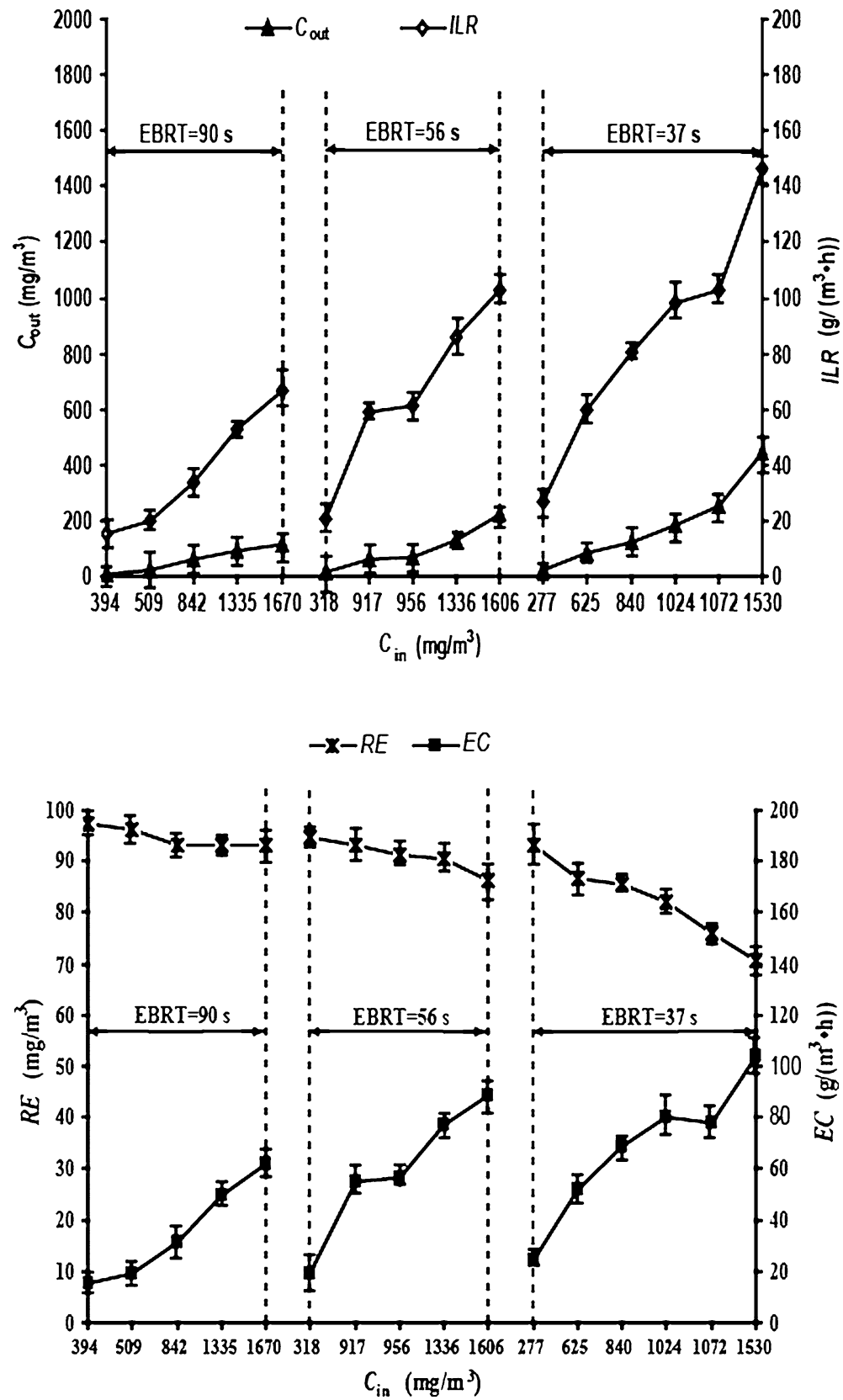

CB levels as affected by the EBRT (empty bed retention time)

EBRT is also an important parameter to control during the operation of the BTF. Mass transfer between the microorganisms and the packing materials is low if the EBRT is too short, and the BTF will operate inefficiently if the EBRT is too long. Given a fixed BTF volume, there is a linear, positive correlation between the EBRT and $Q$. Therefore, the CB levels as a function of EBRT were investigated at EBRTs of 90, $75,56,45$, and $37 \mathrm{~s}$ (corresponding to $Q$ of $0.25,0.3$, $0.4,0.5$, and $0.6 \mathrm{~m}^{3} \mathrm{~h}^{-1}$, respectively), at a constant $v$ of $30 \mathrm{ml} \mathrm{min}^{-1}$ and a $C_{\text {in }}$ of $1,250 \mathrm{mg} \mathrm{m}^{-3}$ (Figs. 6, $7)$.

At a constant $v$, there was a correlation between the EBRT or $Q$ and $C_{\mathrm{in}}, C_{\text {out }}, I L R, E C$ and $R E$. As the EBRT was decreased by increasing $Q, C_{\text {in }}, C_{\text {out }}, I L R$ 
Fig. $6 C_{\text {in }}, C_{\text {out }}$ and $I L R$ at different EBRTs.

$C_{\text {in }}=$ chlorobenzene $(\mathrm{CB})$ gas inlet concentration; $C_{\text {out }}=\mathrm{CB}$ gas outlet concentration; $I L R=\mathrm{CB}$ gas inlet loading rate; $\mathrm{EBRT}=$ empty bed retention time

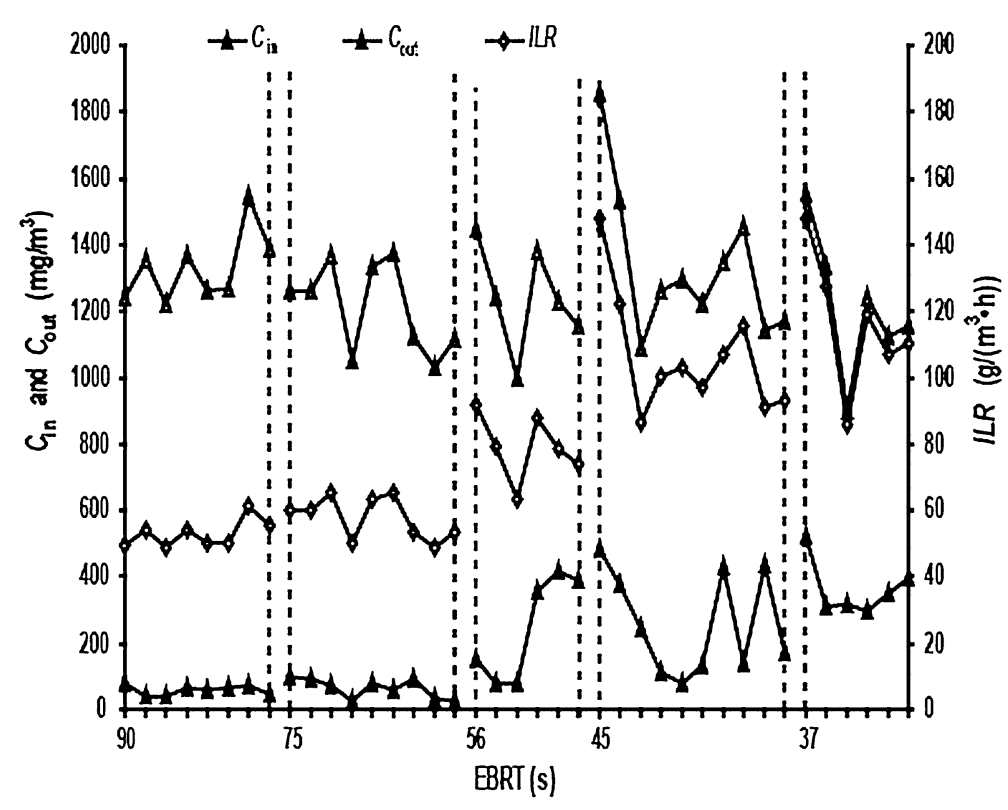

Fig. $7 R E$ and $E C$ at different EBRTs.

$(R E)=$ chlorobenzene $(\mathrm{CB})$ removal efficiency;

$E C=\mathrm{CB}$ elimination capacity; EBRT = empty bed retention time

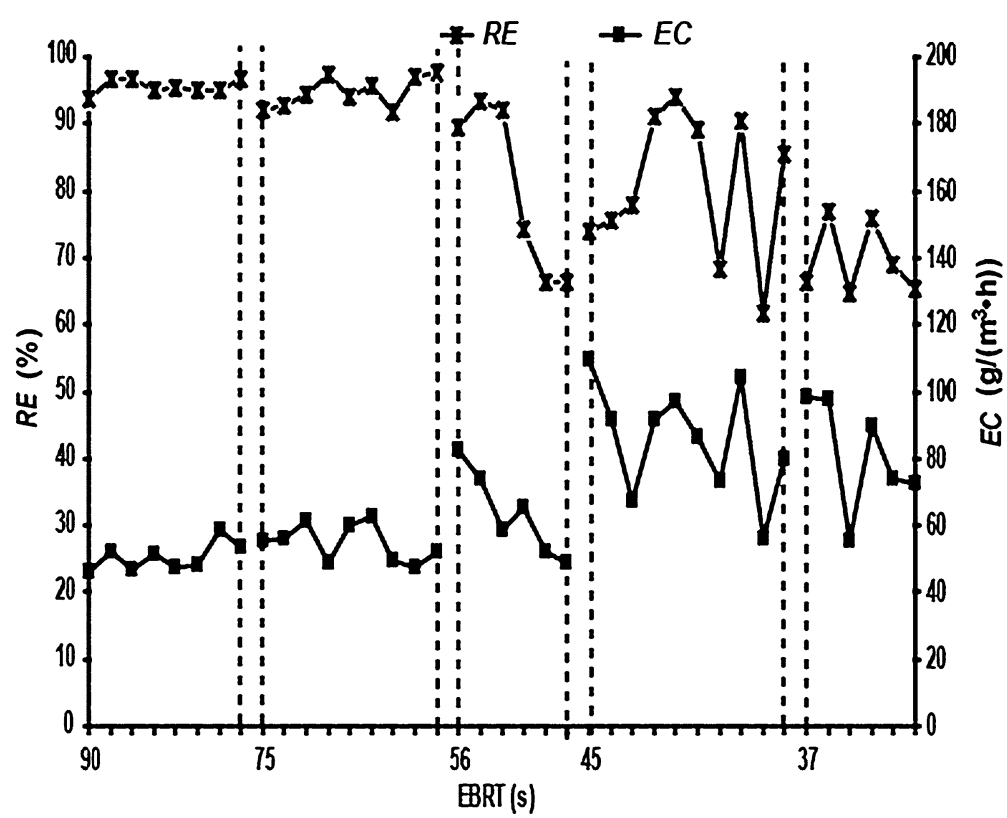

and $E C$ remained constant before increasing. These were all relatively large changes, although the $R E$ was constant at first and then decreased. This shows that the EBRT or $Q$ has a significant impact on the CB levels. At an EBRT of greater than $56 \mathrm{~s}$ (corresponding $Q$ of $<0.4 \mathrm{~m}^{3} \mathrm{~h}^{-1}$ ), $C_{\text {in }}, C_{\text {out }}, I L R, E C$ and $R E$ all fluctuated over a small range. When the EBRT was $<56 \mathrm{~s}$ (or Q exceeded $0.4 \mathrm{~m}^{3} \mathrm{~h}^{-1}$ ), $C_{\text {in }}, C_{\text {out }}, I L R, E C$ and $R E$ severely fluctuated over a large range, particularly at the point where the EBRT or Q was initially changed. This could be due to limitations of the test device, where changes in the EBRT could change $C_{\text {in }}$. When the EBRT was further decreased, $C_{\text {in }}$ was very large, so that the ILR of the BTF was affected by both the EBRT and $C_{\mathrm{in}}$. Therefore, to ensure that $C_{\text {out }}$ meets the CAIES and to improve the operational efficiency of the BTF, the EBRT should be no $<45 \mathrm{~s}$ from a corresponding $Q$ of $<0.5 \mathrm{~m}^{3} \mathrm{~h}^{-1}$. 

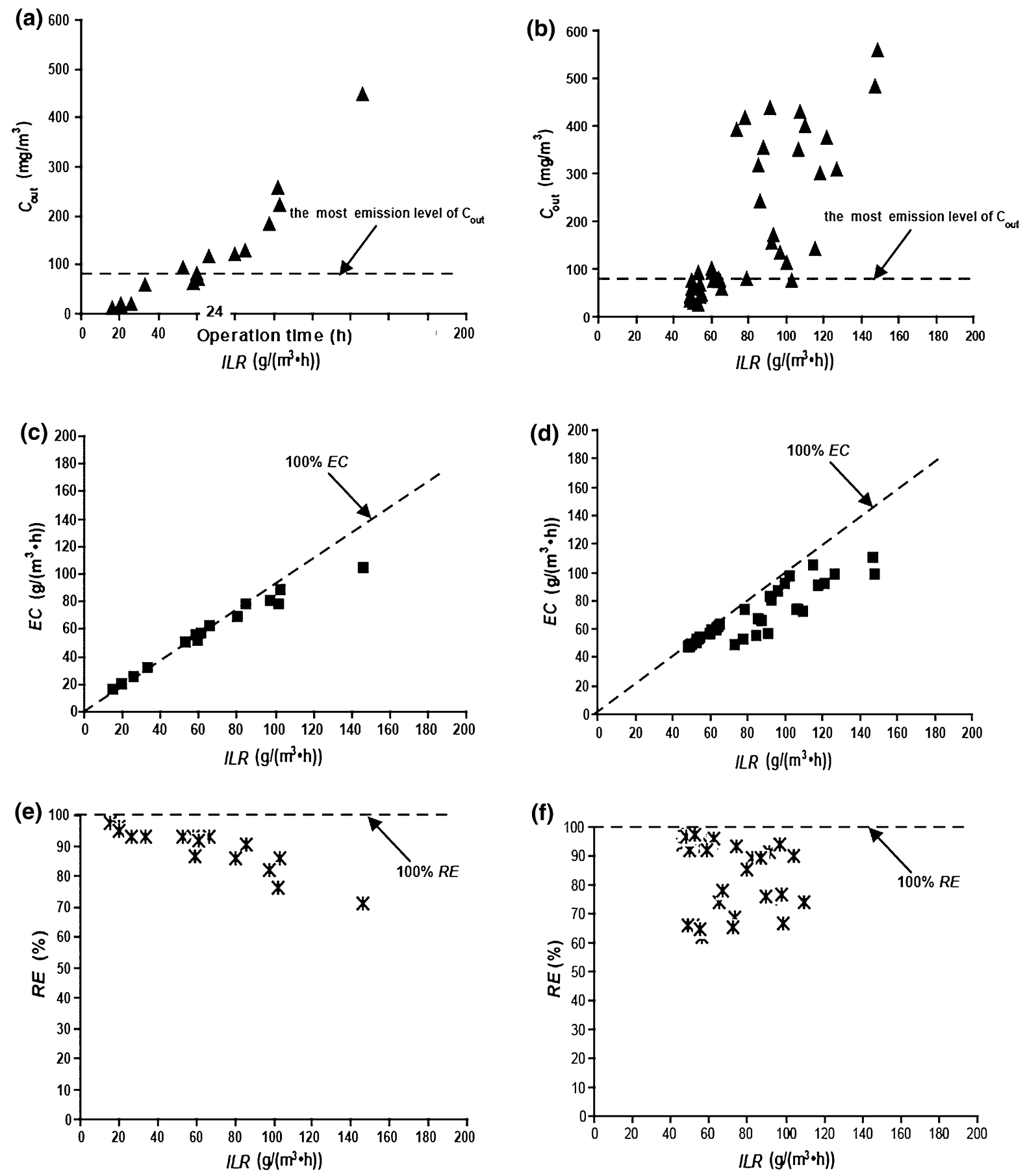

Fig. $8 C_{\text {out }}, E C$ and $R E$ versus $I L R$ a $C_{\text {out }}$ versus $I L R$ when $C_{\text {in }}$ was varied. b $C_{\text {out }}$ versus $I L R$ when the EBRT was varied. c $E C$ versus $I L R$ when $C_{\text {in }}$ was varied. d $E C$ versus $I L R$ when the EBRT was varied. e $R E$ versus $I L R$ when $C_{\text {in }}$ was varied. f $R E$

versus $I L R$ when the EBRT was varied $C_{\text {out }}=$ chlorobenzene (CB) gas outlet concentration; $E C=\mathrm{CB}$ elimination capacity; $R E=\mathrm{CB}$ removal efficiency; $I L R=\mathrm{CB}$ gas inlet loading rate 
CB levels as affected by ILR

Gas purification processes are controlled by the gasliquid mass transfer rate and the biodegradation rate. At low pollutant loads, the biodegradation rate was larger than the mass transfer rate and therefore the process was controlled by the mass transfer rate. However, at high pollutant loads, the biodegradation rate was less than the mass transfer rate, so the process was controlled by the biodegradation rate. ILR depends on both $Q$ and $C_{\text {in }}$. Therefore, a further investigation of the CB levels, as affected by $I L R$, was performed (Fig. 8).

When $I L R$ was increased, $C_{\text {out }}$ and $E C$ increased significantly but $R E$ still decreased. This means that $I L R$ had a more significant impact on the CB levels in the BTF than did $v$. At low ILR, $C_{\text {out }}$ could be maintained to meet the CAIES with a $R E$ of more than $90 \%$ and a linear, positive correlation between $E C$ and $I L R$, which indicated that the mass transfer process and the biodegradation process were working well. When $I L R$ was gradually increased, $C_{\text {out }}$ exceeded this value, and $E C$ and $R E$ increasingly deviated from $100 \%$ removal efficiency. This was probably because the mass transfer process became blocked and the biodegradation process began to play the predominant role. Thus, when the ILR was lower, the degradation of $\mathrm{CB}$ was closer to $100 \%$. When the $I L R$ was higher, the total degradation of $\mathrm{CB}$ was limited by the mass transfer and biodegradation capacity of the BTF. Figure 8 also demonstrates that the distribution of $C_{\text {out }}, E C$ and $R E$ at different EBRTs was more discrete and more disordered than at different $C_{\mathrm{in}}$. This indicated that the EBRT had a greater impact on ILR than $C_{\mathrm{in}}$, which indirectly affected the CB levels of the BTF. To ensure that $C_{\text {out }}$ meets CAIES, the maximum ILR of the BTF can be as high as $103 \mathrm{~g} \mathrm{~m}^{-3} \mathrm{~h}^{-1}$ with a maximum $E C$ of $97 \mathrm{~g} \mathrm{~m}^{-3} \mathrm{~h}^{-1}$ and a maximum $R E$ of $97.7 \%$, by adjusting $v$, the EBRT, and $C_{\text {in }}$.

In conclusion, a BTF with biofilms of the dominant degradation strain, Lysinibacillus fusiformis LW13, stably ran and eliminated significant amounts of CB in the BTF. This study demonstrated a particular advantage in treating high-loading gaseous $\mathrm{CB}$ with this setup as compared with using acclimated sludge (Zhou et al. 2011) or another single dominant species (Zhang et al. 2011). $v$ (Supplementary Fig. 3), $C_{\text {in }}$, the EBRT and ILR affected the CB levels, with the three latter factors displaying the most significant effects. The microorganisms in the BTF adapted to, and resisted, the acidic environment of the spray liquid, while the levels of metabolic intermediates could be monitored and used as a replacement signal for the spray liquid. Of the three indicators, $C_{\text {out }}, E C$ and $R E$, that reflect the BTF's CB purification performance, $C_{\text {out }}$ was the most direct and sensitive.

Acknowledgments This work was financially supported by the Program Fund of Environment Department of Jiangsu Province in China (No. 2012025), the Project Fund for Advancing Industrialization of Scientific Research of Universities in Jiangsu Province in China (No. JHB2012-51), the Innovation Fund for Technology Based Firms of Department of Science and Technology of China (No. 09C26213203714), the Qinglan Program of Science and Technology Innovation Team of Jiangsu Province (2010), and the Research Fund of Key Laboratory for Advanced Technology in Environmental Protection of Jiangsu Province (AE201119).

Conflict of interest The authors declare that they have no conflict of interest.

Open Access This article is distributed under the terms of the Creative Commons Attribution License which permits any use, distribution, and reproduction in any medium, provided the original author(s) and the source are credited.

\section{References}

Field JA, Sierra-Alvarez R (2008) Microbial degradation of chlorinated benzenes. Biodegradation 19:463-480

Huang YL, Liu T, Wang C, Wang JF (2013) Mechanism and kinetics of the synthesis of phenyltrichlorosilane from trichlorosilane and chlorobenzene by gas phase condensation. Chem Eng J 226:255-262

Lebrero R, Estrada JM, Muñoz R, Quijano G (2012) Toluene mass transfer characterization in a biotrickling filter. Biochem Eng J 60:44-49

Li ZX, Niu X, He WY, Tong YY, Jin H, Ding C (2013) Screening of chlorobenzene-degrading bacterium and purification of its degradation enzyme (in Chinese). Wei Sheng Wu Xue Bao 53:455-463

Liu JW, Wang ZL (2012) Research on packing materials selection for biotrickling filter to treat waste gas containing organic compounds. Environ Pollut Control 34:17-21

Liu XJ, Wang XY, Zhang ZF, Li YY (2011) Enhancement effect of surfactant on purifying chlorobenzene waste gas by liquid absorption-stripping. J North Univ China (Nat Sci Edn) 32:723-726

Lopez ME, Rene ER, Malhautier L, Rocher J, Bayle S, Veiga MC, Kennes C (2013) One-stage biotrickling filter for the removal of a mixture of volatile pollutants from air: performance and microbial community analysis. Bioresour Technol 138:245-252 
Nicolella C, Converti A, Zilli M (2009) Biotrickling air filtration of 2-chlorophenol at high loading rates. Biochem Eng J 43:98-105

Seignez C, Atti A, Adler N, Péringer P (2002) Effect of biotrickling filter operating parameters on chlorobenzenes degradation. J Environ Eng 128:360-366

Xue NT, Wang QH, Wu CF, Zhang LH, Xie WM (2010) Enhanced removal of $\mathrm{NH}_{3}$ during composting by a biotrickling filter inoculated with nitrifying bacteria. Biochem Eng J 51:86-93

Yang C, Chen H, Zeng G, Yu G, Luo S (2010) Biomass accumulation and control strategies in gas biofiltration. Biotechnol Adv 28:531-540

Zhang LF, Anderson WA (2013) Effect of ozone and sulfur dioxide on the photolytic degradation of chlorobenzene in air. Ind Eng Chem Res 52:3315-3319
Zhang LL, Leng SQ, Zhu RY, Chen JM (2011) Degradation of chlorobenzene by strain Ralstonia pickettii L2 isolated from a biotrickling filter treating a chlorobenzene-contaminated gas stream. Appl Microbiol Biotechnol 91:407-415

Zhao XK, Yang GP, Wu P, Li NH (2001) Study on adsorption of chlorobenzene on marine sediment. J Coll Interface Sci 243:273-279

Zhou QW, Zhu RY, Hu J, Zhang LL, Chen JM. (2011) BTF performance treating a chlorobenzene-contaminated gas stream (in Chinese). Huan jing ke xue = Huanjing kexue/ [bian ji, Zhongguo ke xue yuan huan jing ke xue wei yuan hui "Huan jing ke xue" bian ji wei yuan hui] 32:3673-3679 